\title{
Attitudes of Patients with Cutaneous Melanoma Towards Prognostic Testing Using Gene Expression Profiling
}

Lorrie-Beth Miley ${ }^{1}$, Kelli Ahmed PhD², Jennifer J. Siegel PhD², Sonia K. Morgan-Linnell PhD²,

Kyleigh LiPira ${ }^{1}$

1.Melanoma Research Foundation; Washington, DC, USA; 2. Castle Biosciences, Inc. Friendswood, Texas

\section{Background}

The 31-gene expression profile (31-GEP) test for cutaneous melanoma (CM) assesses gene expression measurements from formalin-fixed paraffin-embedded primary tumor tissue to predict risk of tumor recurrence or metastasis. 2The 31-GEP stratifies risk into one of three risk categories: low risk (Class 1A), multiple prospective and retrospective studies ${ }^{1-7}$.

\section{Objective}

IUnderstand patients' perspectives on prognostic and 31-GEP testing and whether patients experience decision regret after having 31-GEP testing performed.

\section{Methods}

IA 43-question online survey was distributed by the Melanoma Research Wrom June 14, 2021, through August 2, 2021 .

>Patients were asked a series of five validated questions that gauge patients' level of regret regarding the decision to undergo 31-GEP testing and the extent to which they experienced decision regret. being no regret, 5 being high regret, and 3 neutral. Responses were limited to 31-GEP prepnostic testing became avalable:

\section{Results}

\section{Table 1. Participant Demographics}

\section{Demographics ( $n=120)$}

Male

Female

Prefer not to share
Did you have DecisionDx testing?

Yes
No

Unsure

Commercial with MedAdvantage

Medicare
None
I don't know
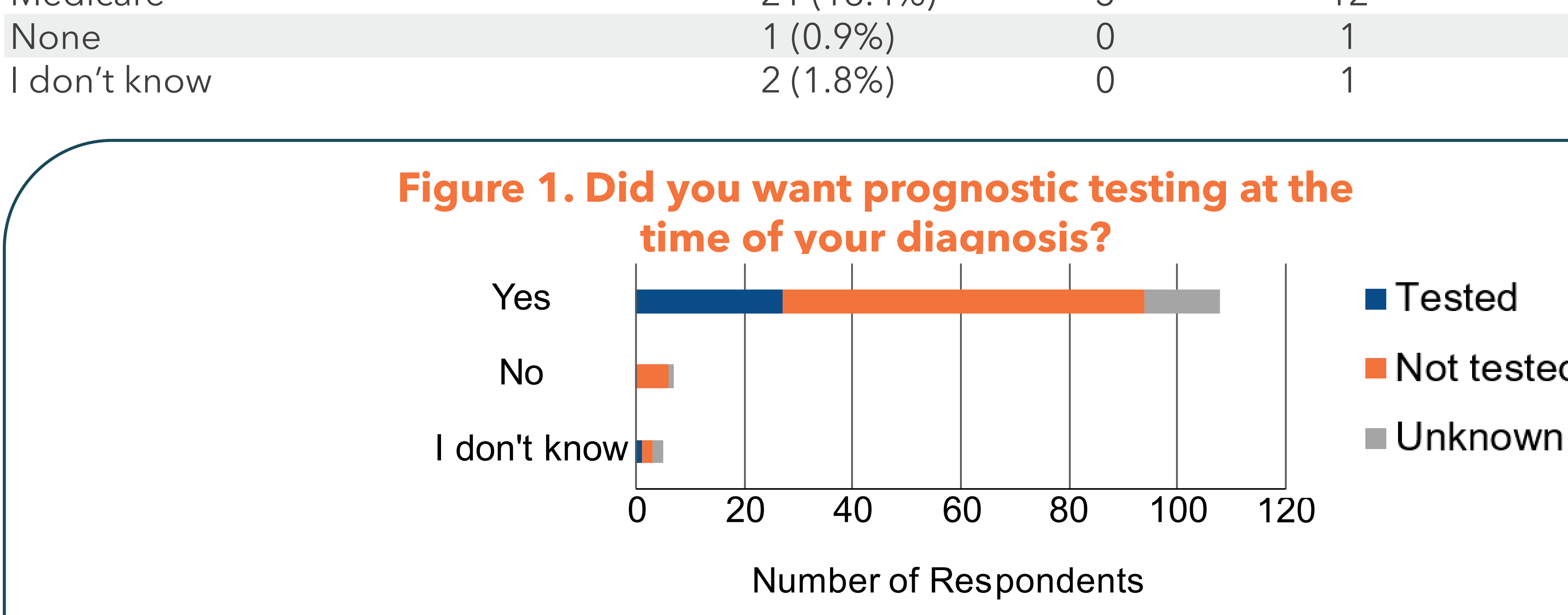

Respondents were asked if they wanted prognostic information about their GEP or not, desired prognostic testing about their tumor.

\section{Results}

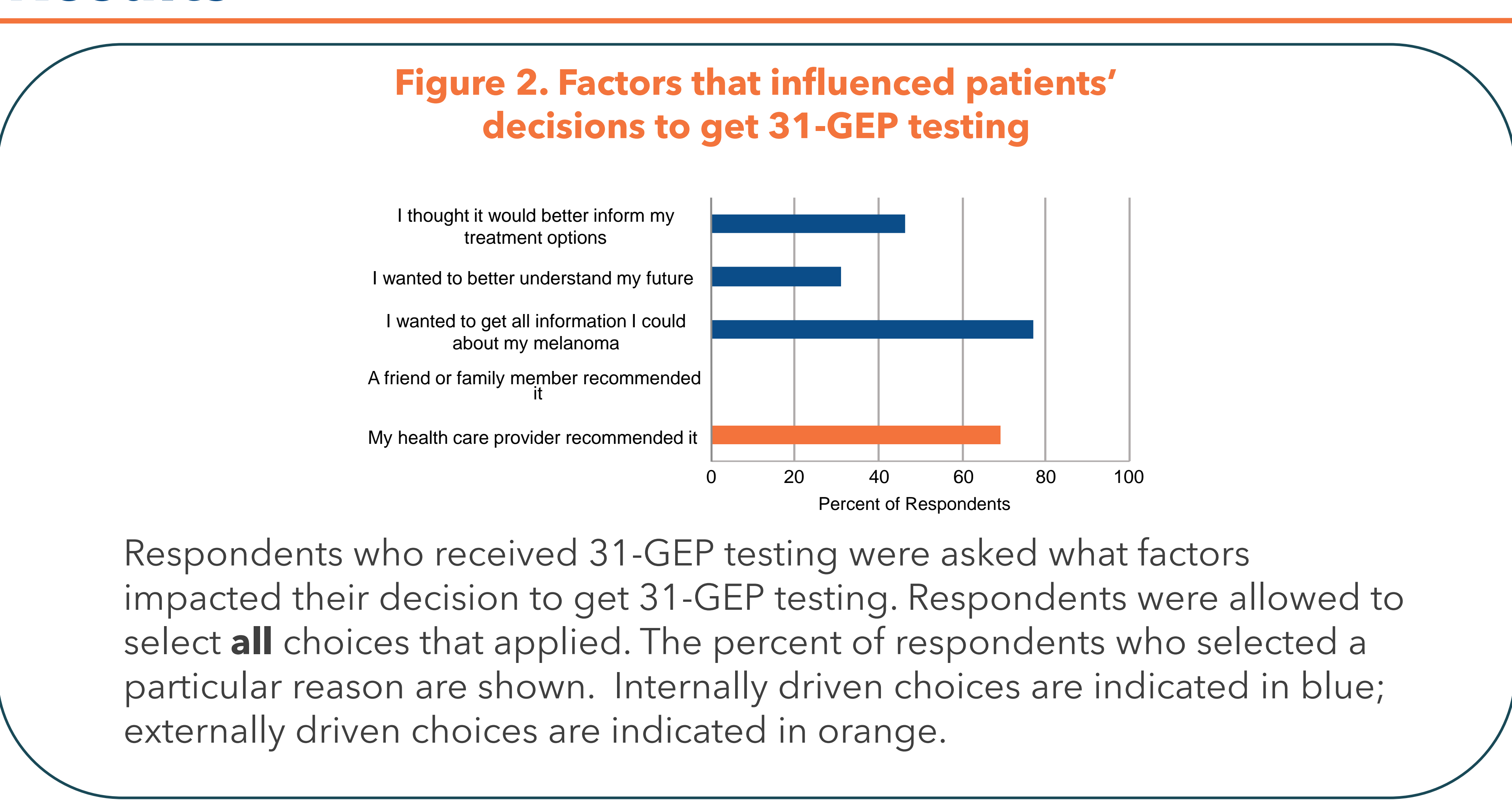

Figure 3. Utility of 31-GEP test information

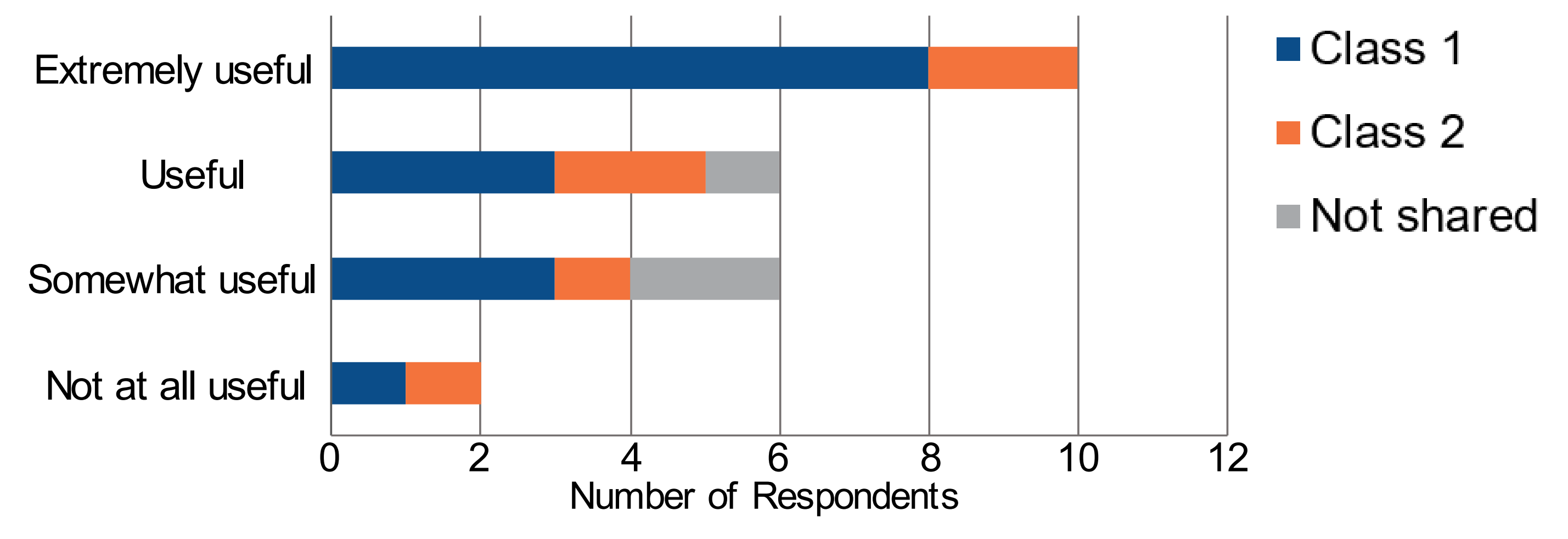

Respondents who received $31-G E P$ testing $(n=24$ responses) answered
whether they felt that the results were useful. The graph indicates the number of respondents who chose a particular choice and their reported 31-GEP class call. Most patients thought the 31-GEP was at least somewhat useful.

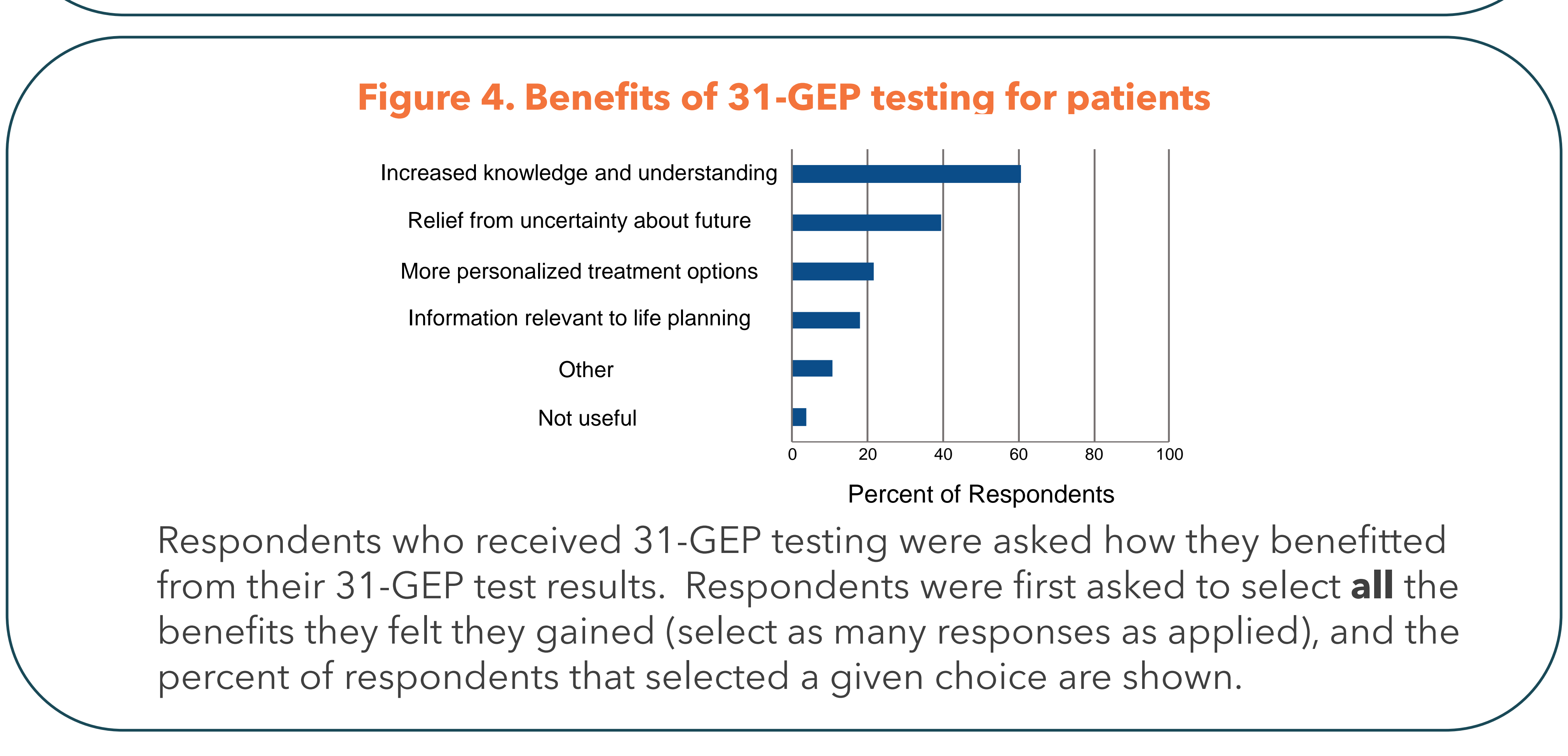

Participants who received 31-GEP testing felt that the results were useful to them Testing game them increased knowledge, relief from uncertainty, personalized treatment options, and information for life planning.

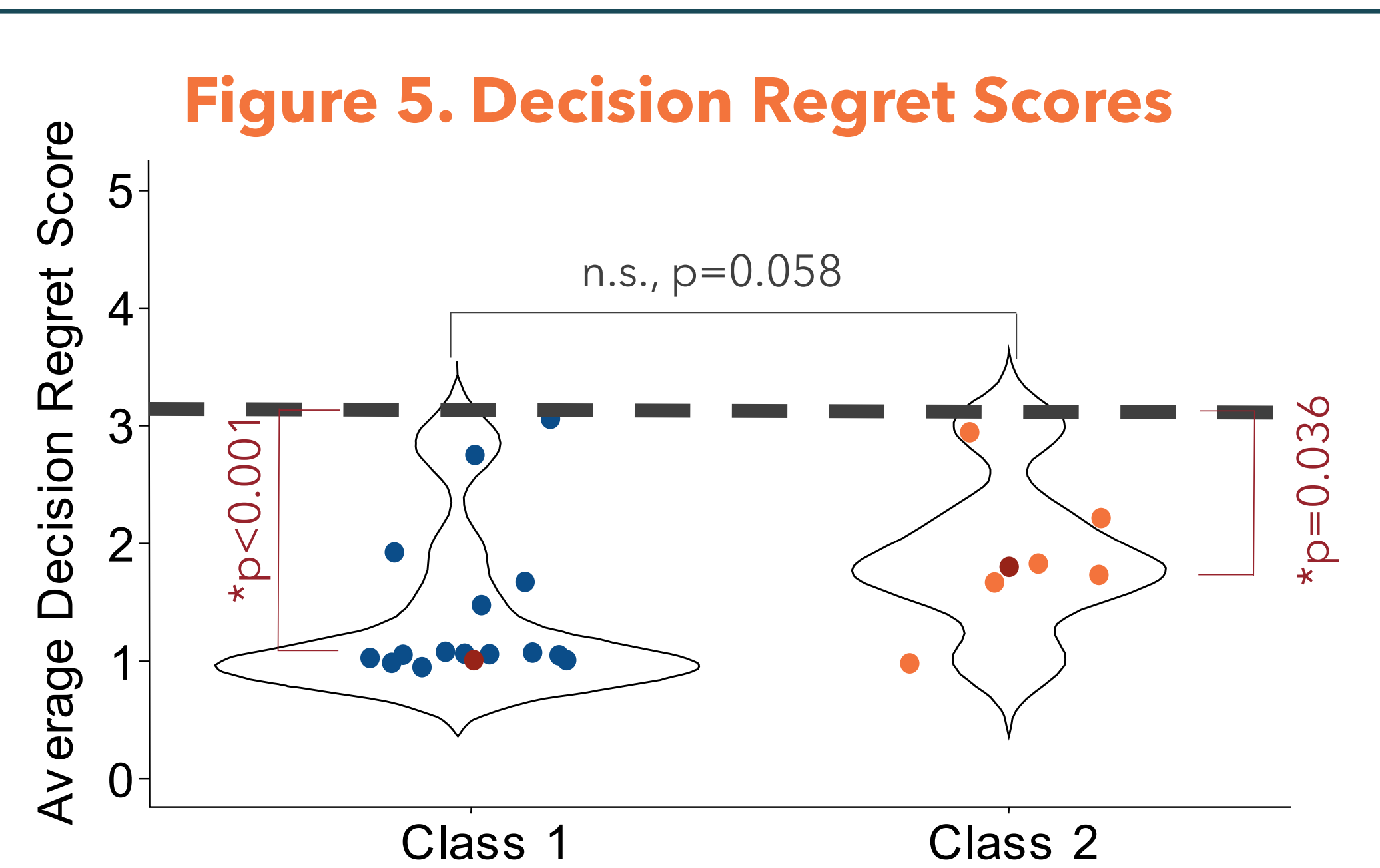

Respondents were asked a validated series questions regarding the level of regret orange (Class 2) circles represent the mean decision regret score for each respondent. The dashed line indicates decision regret (3.05). Median decision regret different $(p=0.058)$. Both Class $1(p<0.001)$ and Class $2(p=0.036)$ median de (e) regret regardless of high or low risk results. *, statistically significant: $n . s$, not significant

\section{Conclusions}

$90 \%$ of patients wanted prognostic information about their tumors at the time of diagnosis.

Patients wanted 31-GEP testing to increase their knowledge about their disease $(76.9 \%)$ and inform treatment decisions $(46.2 \%)$.

Patients (>90\%) felt 31-GEP testing was useful and felt they gained understanding $(60.7 \%)$ and relief from uncertain $(39.3 \%)$.

Patients receiving 31-GEP results did not experience decision regret, even among patients who had Class 2 , high-risk tumors

\section{References}

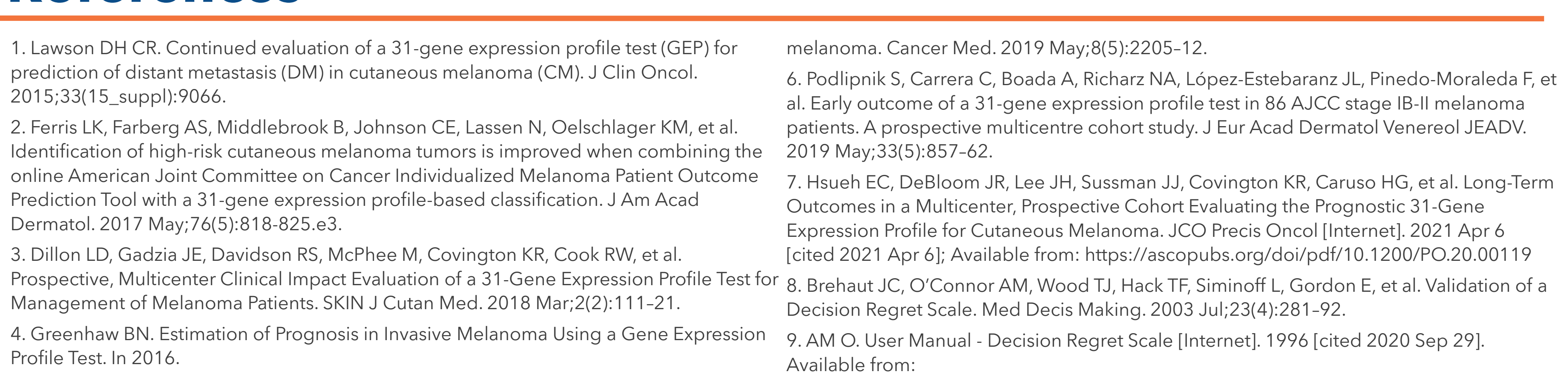

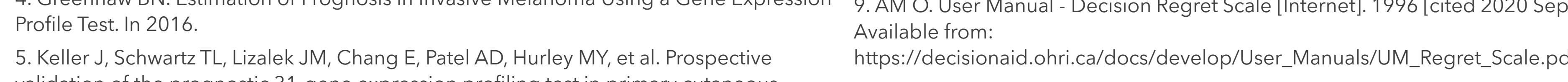

Acknowledgments \& Disclosures

; This study was sponsorede by Caste Biosciencess. Inc. 